\title{
Characterization of Craniocervical Artery Dissection by Simultaneous MR Noncontrast Angiography and Intraplaque Hemorrhage Imaging at 3T
}

\author{
Q. Li, J. Wang, H. Chen, X. Gong, N. Ma, K. Gao, L. He, M. Guan, Z. Chen, R. Li, D. Mi, C. Yuan, X. Zhao, and X.H. Zhao
}

\begin{abstract}
BACKGROUND AND PURPOSE: Craniocervical artery dissection is the most common cause of ischemic stroke identified in young adults. For the diagnosis of craniocervical artery dissection, multisequence MR imaging is recommended but is time-consuming. Recently, investigators proposed a simultaneous noncontrast angiography and intraplaque hemorrhage imaging technique allowing simultaneous noncontrast MRA and vessel wall imaging in a single scan. This study sought to investigate the feasibility of 3D simultaneous noncontrast angiography and intraplaque hemorrhage MR imaging in the characterization of craniocervical artery dissection.
\end{abstract}

MATERIALS AND METHODS: Twenty-four symptomatic patients (mean age, $45.0 \pm 16.1$ years; 21 men) with suspected craniocervical artery dissection were recruited. The 3D simultaneous noncontrast angiography and intraplaque hemorrhage 3D TOF MRA and blackblood imaging sequences were performed on a 3T MR imaging scanner. The agreement between simultaneous noncontrast angiography and intraplaque hemorrhage imaging and multisequence MR imaging in evaluating arterial dissection was determined.

RESULTS: Dissection was found to involve 1 artery in 22 patients and 2 arteries in 2 patients. The intramural hematoma and luminal occlusion were detected in 19 (79.2\%) and 11 (45.8\%) patients, respectively. In measuring stenosis, the Cohen $\kappa$ value between 3D TOF MRA and simultaneous noncontrast angiography and intraplaque hemorrhage imaging was $0.82(P<.001)$. All intramural hematomas on multisequence imaging were successfully identified by simultaneous noncontrast angiography and intraplaque hemorrhage imaging.

CONCLUSIONS: 3D simultaneous noncontrast angiography and intraplaque hemorrhage imaging showed excellent agreement with multisequence MR imaging in evaluating luminal stenosis and intramural hematoma in patients with craniocervical artery dissection. The simultaneous noncontrast angiography and intraplaque hemorrhage imaging saved nearly $50 \%$ of scanning time compared with multisequence MR imaging. Our findings suggest that 3D simultaneous noncontrast angiography and intraplaque hemorrhage imaging might be an alternative, time-efficient diagnostic tool for craniocervical artery dissection.

ABBREVIATIONS: $C C A D=$ craniocervical artery dissection; $I M H=$ intramural hematoma; $M E R G E=$ Multi-Echo Recombined Gradient Echo; SNAP $=$ simultaneous noncontrast angiography and intraplaque hemorrhage

$\mathrm{C}$ raniocervical artery dissection (CCAD) is the most common cause of stroke identified in young and middle-aged adults. ${ }^{1,2}$ Pathophysiologically, arterial dissections occur when a tear develops

Received October 30, 2014; accepted after revision February 13, 2015.

From the Departments of Neurology (Q.L., X.G., D.M., X.Z.) and Interventional Neuroradiology (N.M., K.G.), Beijing Tiantan Hospital, Capital Medical University, Beijing, China; Department of Neurology (Q.L.), People's Hospital of Xinjiang Uygur Autonomous Region, Urumqi, China; Clinical Sites Research Program (J.W.), Philips Research North America, Briarcliff Manor, New York; Department of Biomedical Engineering (H.C., L.H., Z.C., R.L., C.Y., X.H.Z.), Center for Biomedical Imaging Research, Tsinghua University School of Medicine, Beijing, China; Department of Radiology (M.G.), Yangzhou First People's Hospital, Yangzhou, China; and Department of Radiology (C.Y.), University of Washington, Seattle, Washington.

This study was supported in part by a grant from the National Natural Science Foundation of China (81271536), a grant from the Beijing Municipal Science and Technology Commission (D111107003111007), and Philips Healthcare.

Drs Xi Hai Zhao and Xingquan Zhao are co-corresponding authors who contributed equally to this study. in $\geq 1$ layer of the vessel wall, allowing blood to enter the wall and split the layers; this condition is characterized by a cavity or intramural hematoma (IMH). ${ }^{3}$ The increased volume of the IMH may narrow or occlude the lumen, and it can also damage the intima and trigger thrombosis. Because this arterial disorder is strongly associated with acute ischemic events, ${ }^{4,5}$ it is clinically preferable to diagnose CCAD at the early stages so that clinicians can properly treat and prevent potential neurologic complications. ${ }^{6}$

\footnotetext{
Paper previously presented in part at: Annual International Workshop on Magnetic Resonance Angiography, August 20-23, 2013; New York, New York.

Please address correspondence to Xi Hai Zhao, MD, PhD, Center for Biomedical Imaging Research, Tsinghua University School of Medicine, Haidian District, 100084 Beijing, China; e-mail: xihaizhao@tsinghua.edu.cn; and Xingquan Zhao, MD, PhD, Department of Neurology, Beijing Tiantan Hospital, Capital Medical University, No. 6 Tiantanxili, Dongcheng District, 100050, Beijing, China; e-mail: zxq@vip.163.com - Indicates open access to non-subscribers at www.ajnr.org

http://dx.doi.org/10.3174/ajnr.A4348
}

AJNR Am J Neuroradiol 36:1769-75 Sep 2015 www.ajnr.org 
Angiographic imaging modalities, such as CTA, MRA, and DSA, have been widely used for the diagnosis of CCAD in clinical settings. ${ }^{7-9}$ These approaches can only provide the information of luminal narrowing or occlusion but are unable to directly delineate IMH, which is a key sign of CCAD. Some investigators believe that craniocervical arteries with occlusion but without evidence of IMH cannot be diagnosed as dissection unless the dissected wall is completely recanalized. ${ }^{10} \mathrm{MR}$ vessel wall imaging has been increasingly used for the diagnosis of CCAD due to its capability of directly visualizing IMH in the vessel wall. ${ }^{11-13}$ Recently, a multisequence MR imaging protocol, which includes both MRA and MR vessel wall imaging sequences, was recommended for the diagnosis of CCAD. ${ }^{10} \mathrm{How}-$ ever, this protocol needs independent acquisition of 2 different timeconsuming MR imaging sequences.

Most recently, a simultaneous noncontrast angiography and intraplaque hemorrhage (SNAP) MR imaging technique has been proposed for the evaluation of vulnerable carotid atherosclerotic plaques. ${ }^{14}$ The major advantage of the 3D SNAP imaging sequence is acquiring noncontrast MRA and vessel wall images simultaneously in a single scan. The vessel wall images derived from the 3D SNAP sequence carry heavy T1-weighting, which is very sensitive to intraplaque hemorrhage or IMH. The other advantages of SNAP imaging include fast scanning, large longitudinal coverage (up to $250 \mathrm{~mm}$ ), and isotropic high resolution $(0.8$ $\mathrm{mm}^{3}$ ). Those advantages make SNAP an ideal candidate for quick and reliable identification of dissection, particularly for tortuous craniocervical arteries with longer lesions.

We hypothesized that the 3D SNAP MR imaging technique is capable of characterizing CCAD by providing both luminal and vessel wall information simultaneously. In this study, we sought to evaluate the feasibility of 3D SNAP MR imaging in the diagnosis of CCAD.

\section{MATERIALS AND METHODS Participants}

Patients with recent ischemic cerebrovascular symptoms (within 2 weeks) and with suspected craniocervical artery dissection were prospectively and consecutively recruited in this study. The inclusion criteria were the following: 1) recent ischemic symptoms and/or neck pain and/or headache; 2) MR angiography revealing stenosis with a string sign or total occlusion or a double lumen or luminal dilation (pseudoaneurysm). ${ }^{15}$ The exclusion criteria were as follows: 1 ) subjects with evidence of cardiogenic ischemic stroke, 2) subjects with evidence of cerebrovascular atherosclerotic disease according to traditional imaging examinations, and 3) subjects with contraindications to MR imaging. The clinical characteristics, including age, sex, hypertension, diabetes, smoking, and hyperlipidemia, were collected. The study protocol was approved by the local institutional review board before the initiation of this study, and written consent forms were obtained from all subjects.

\section{MR Imaging}

All subjects underwent MR imaging on a 3T Achieva TX scanner (Philips Healthcare, Best, the Netherlands) with a custom-designed 36-channel neurovascular coil. ${ }^{16}$ The multisequence MR imaging, including 3D TOF MRA and 3D Multi-Echo Recombined Gradient Echo (MERGE; GE Healthcare, Milwaukee, Wis- consin), was performed with the following parameters: $3 \mathrm{D}$ TOF MRA, turbo field echo; TR/TE, 25/3.5 ms; flip angle, $20^{\circ}$; FOV, $180 \times 180 \times 84 \mathrm{~mm}^{3}$ for intracranial arteries and $130 \times 168 \times$ $140 \mathrm{~mm}^{3}$ for carotid arteries; voxel size, $0.3 \times 0.3 \times 0.7 \mathrm{~mm}^{3}$ for intracranial arteries and $0.6 \times 0.6 \times 0.9 \mathrm{~mm}^{3}$ for carotid arteries; total scanning time, 9 minutes; 3D MERGE, turbo field echo; TR/TE, 9.2/4.3 ms; flip angle, $6^{\circ}$; FOV, $250 \times 160 \times 40 \mathrm{~mm}^{3}$; scanning time, 4 minutes 6 seconds. In this study, the presence of IMH was determined by 3D MERGE images when lesions appeared hyperintense because the imaging sequence MERGE has T1 contrast-weighting and has the potential to identify intraplaque hemorrhage. ${ }^{17}$ In contrast, the 3D SNAP imaging sequence was acquired with the following parameters: $3 \mathrm{D}$ SNAP, turbo field echo sequence; TR/TE, $10 / 4.8 \mathrm{~ms}$; flip angle, $11^{\circ} / 5^{\circ}$; FOV, $250 \times 160 \times 32 \mathrm{~mm}^{3}$; voxel size, $0.8 \times 0.8 \times 0.8 \mathrm{~mm}^{3}$; scanning time, 6 minutes 45 seconds. The 3D SNAP and MERGE images were acquired in the coronal direction, and 3D TOF MRA images were acquired in the axial direction. Both extracranial carotid arteries and intracranial arteries were covered on MR imaging.

\section{Image Interpretation}

The 3D TOF MRA, MERGE, and SNAP images were reconstructed by using maximum intensity projection, minimum intensity projection, and MPR at the workstation Extended MR WorkSpace (Version 2.0.3.3; Philips Healthcare). A color-coded algorithm was used to create $3 \mathrm{D}$ maximum intensity projection images from SNAP images for joint display of luminal changes and the components with hyperintense signal in the vessel wall. ${ }^{14}$ Blinded to clinical information and SNAP images, 2 reviewers evaluated with consensus the degree of luminal stenosis on $3 \mathrm{D}$ TOF MRA images and the presence or absence of IMH, which is defined as hyperintense signal in the vessel wall on 3D MERGE images. The diagnosis of IMH was made when the signal intensity of the vessel wall was 2 times as high as the reference tissue of muscle. The other 2 reviewers measured the luminal stenosis on noncontrast MRA derived from SNAP images and identified the presence or absence of IMH on vessel wall images from SNAP imaging blinded to clinical information and 3D TOF MRA and MERGE image findings. Luminal stenosis was measured by using the NASCET method. ${ }^{18}$ All observers were neuroradiologists and had $>3$ years' experience in cerebrovascular imaging. The luminal stenosis was divided into the following categories: mild stenosis $(<50 \%)$, moderate stenosis $(50 \%-70 \%)$, severe stenosis (70\%-99\%), and occlusion (100\%). The presence or absence of pseudoaneurysm, double lumen and intimal flaps, and lesion length was also assessed.

\section{Statistical Analysis}

The continuous variables were described as mean $\pm \mathrm{SD}$, and categoric ones were presented as absolute frequencies or percentages. The Cohen $\kappa$ was used for evaluating the agreement between 3D TOF MRA and SNAP imaging in measuring luminal stenosis. The agreement between 3D SNAP and 3D MERGE in the identification of IMH, pseudoaneurysm, intimal flaps, and double lumen was also determined. A $P$ value of $<.05$ was statistically significant. All statistical analyses were conducted in SPSS 19.0 (IBM, Armonk, New York). 


\section{RESULTS}

In total, 24 patients (mean age, $45.0 \pm 16.1$ years; 21 men) were recruited for this study between June 2012 and September 2014. Of the 24 patients, 4 (16.7\%) presented with headache, $3(12.5 \%)$ presented with neck pain, $20(83.3 \%)$ had ischemic stroke, and 4 $(16.7 \%)$ had TIA. All patients denied blunt traumatic injury to the head and neck. The clinical characteristics are summarized in Table 1.

\section{Characteristics on Multisequence 3D MR Imaging}

The characteristics of each subject on multisequence MR images are shown in Table 2. Twenty-two patients developed dissection in 1 artery, and 2 patients had dissection in 2 arteries. The dissection involvement in different vascular beds was as follows: 3

Table 1: Clinical characteristics of study population

\begin{tabular}{lcc}
\hline \multicolumn{1}{c}{ Characteristics } & $\begin{array}{c}\text { Mean or No. } \\
\text { (\%) }\end{array}$ & $\begin{array}{c}\text { Range } \\
\text { (if Applicable) }\end{array}$ \\
\hline Age (yr) & $45.0 \pm 16.1$ & $14-70$ \\
Male sex & $21(87.5)$ & \\
Weight (kg) & $75.1 \pm 8.5$ & $60-90$ \\
Height (cm) & $171.2 \pm 5.5$ & $160-183$ \\
Smoking & $10(41.7)$ & \\
Hypertension & $13(54.2)$ & \\
Diabetes & $6(25)$ & \\
High-density lipoprotein (mmol/L) & $1.0 \pm 0.2$ & $0.5-1.8$ \\
Low-density lipoprotein (mmol/L) & $2.3 \pm 0.8$ & $1.0-5.7$ \\
Total cholesterol (mmol/L) & $4.0 \pm 1.1$ & $2.2-7.8$ \\
Triglycerides (mmol/L) & $1.5 \pm 0.7$ & $0.6-3.0$ \\
Headache & $4(16.7)$ & \\
Neck pain & $3(12.5)$ & \\
Ischemic stroke & $20(83.3)$ & \\
Transient ischemic attack & $4(16.7)$ & \\
\hline
\end{tabular}

$(12.5 \%)$ in the common carotid artery, $8(33.3 \%)$ in the $\mathrm{C} 1 \mathrm{seg}$ ment of the internal carotid arteries, $3(12.5 \%)$ in the middle cerebral artery, $6(25 \%)$ in the basilar artery, and $6(25 \%)$ in the vertebral artery. IMHs were detected in 19 (79.2\%) patients. In arteries with dissection, we found no luminal changes (stenosis or dilation) in $3(12.5 \%)$ patients, mild stenosis in 2 (8.3\%) patients, moderate stenosis in $3(12.5 \%)$ patients, severe stenosis in 3 $(12.5 \%)$ patients, and occlusion in $11(45.8 \%)$ patients. The pattern of pseudoaneurysm and intimal flaps and double lumen was observed in $2(8.3 \%)$ and $3(16.7 \%)$ patients, respectively.

\section{Agreement between 3D SNAP and Multisequence 3D MR Imaging}

In measuring luminal stenosis of diseased arteries, the Cohen $\kappa$ value between 3D TOF MRA and SNAP imaging was $0.82(P<$ $.001)$. Figure 1 demonstrates mild stenosis in the basilar artery on the MRA image derived from SNAP imaging (Fig 1A), corresponding to the TOF MRA image (Fig $1 B$ ), while the hyperintense signal on the SNAP vessel wall image (Fig $1 C$ ) indicates IMH. One patient with mild stenosis on 3D TOF MRA was classified as having moderate stenosis by SNAP imaging, and 2 patients with severe stenosis on 3D TOF MRA were diagnosed as having occlusion. All IMHs on multisequence imaging were successfully identified by SNAP imaging. IMH appeared hyperintense on the 3D SNAP image, corresponding to the 3D MERGE images (Fig 2). In addition, the characteristics of pseudoaneurysm, intimal flaps, and double lumen on multisequence imaging were also accurately determined by SNAP imaging (Fig 3). Figure 4 is an example of the excellent joint visualization of luminal changes and IMH in the vessel wall on SNAP images after color-coded reconstruction.

Table 2: Characteristics of 24 patients with CCAD on multisequence MR imaging

\begin{tabular}{|c|c|c|c|c|c|c|c|}
\hline \multirow[b]{2}{*}{$\begin{array}{l}\text { Patient } \\
\text { No. }\end{array}$} & \multirow[b]{2}{*}{$\begin{array}{l}\text { Sex/Age } \\
\text { (yr) }\end{array}$} & \multirow[b]{2}{*}{ Location } & \multirow[b]{2}{*}{$\begin{array}{l}\text { Length } \\
(\mathrm{mm})\end{array}$} & \multirow[b]{2}{*}{$\begin{array}{l}\text { Category of } \\
\text { Stenosis }^{\mathrm{a}}\end{array}$} & \multicolumn{3}{|c|}{ Presence or Absence } \\
\hline & & & & & IMH & Pseudo-aneurysm & $\begin{array}{l}\text { Intimal Flaps and } \\
\text { Double Lumen }\end{array}$ \\
\hline 1 & $M / 65$ & BA & 16.7 & Moderate & + & - & - \\
\hline 2 & $M / 24$ & MCA & 8.1 & Occlusion & + & - & - \\
\hline 3 & $M / 14$ & MCA & 6.0 & Mild & + & - & - \\
\hline 4 & $M / 53$ & $\mathrm{ICA}(\mathrm{Cl})$ & 35.8 & No stenosis & - & + & + \\
\hline 5 & $M / 20$ & BA & 12.7 & No stenosis & - & - & + \\
\hline 6 & $M / 40$ & $\mathrm{ICA}(\mathrm{Cl})$ & 70.2 & Occlusion & + & - & - \\
\hline 7 & $M / 62$ & $\mathrm{BA}$ and $\mathrm{VA}$ & 44.1 & Occlusion & + & - & - \\
\hline 8 & $M / 59$ & BA & 22.2 & No stenosis & - & + & - \\
\hline 9 & $M / 36$ & MCA & 10.7 & No stenosis & - & - & + \\
\hline 10 & $\mathrm{~F} / 18$ & $\mathrm{CCA}$ & 23.3 & Occlusion & + & - & - \\
\hline 11 & $M / 34$ & VA & 10.1 & Moderate & - & - & - \\
\hline 12 & $\mathrm{~F} / 38$ & $\mathrm{ICA}(\mathrm{Cl})$ & 42.4 & Severe & + & - & - \\
\hline 13 & $\mathrm{~F} / 66$ & CCA & 44.8 & No stenosis & + & - & - \\
\hline 14 & $M / 43$ & VA & 7.5 & Occlusion & + & - & - \\
\hline 15 & $M / 62$ & $\mathrm{ICA}(\mathrm{Cl})$ & 40 & Severe & + & - & - \\
\hline 16 & $M / 68$ & $C C A$ & 37.1 & Occlusion & + & - & - \\
\hline 17 & $M / 42$ & VA & 24.8 & Occlusion & + & - & - \\
\hline 18 & $M / 35$ & $\mathrm{ICA}(\mathrm{Cl})$ & 63.4 & Occlusion & + & - & - \\
\hline 19 & $M / 51$ & $\mathrm{BA}$ and $\mathrm{VA}$ & 50.8 & Mild & + & - & - \\
\hline 20 & $M / 69$ & $\mathrm{ICA}(\mathrm{Cl})$ & 57 & Occlusion & + & - & - \\
\hline 21 & $M / 56$ & ICA (CI) & 66.4 & Severe & + & - & - \\
\hline 22 & $M / 40$ & ICA (CI) & 13.6 & Occlusion & + & - & - \\
\hline 23 & $M / 41$ & VA & 42.7 & Occlusion & + & - & - \\
\hline 24 & $M / 43$ & BA & 11.6 & Moderate & + & - & + \\
\hline
\end{tabular}

Note:-BA indicates basilar artery; VA, vertebral artery; CCA, common carotid artery; +, positive; -, negative

${ }^{a}$ Mild stenosis, $1 \%-49 \%$; moderate stenosis, 50\%-69\%; severe stenosis, $70 \%-99 \%$; occlusion, $100 \%$. 

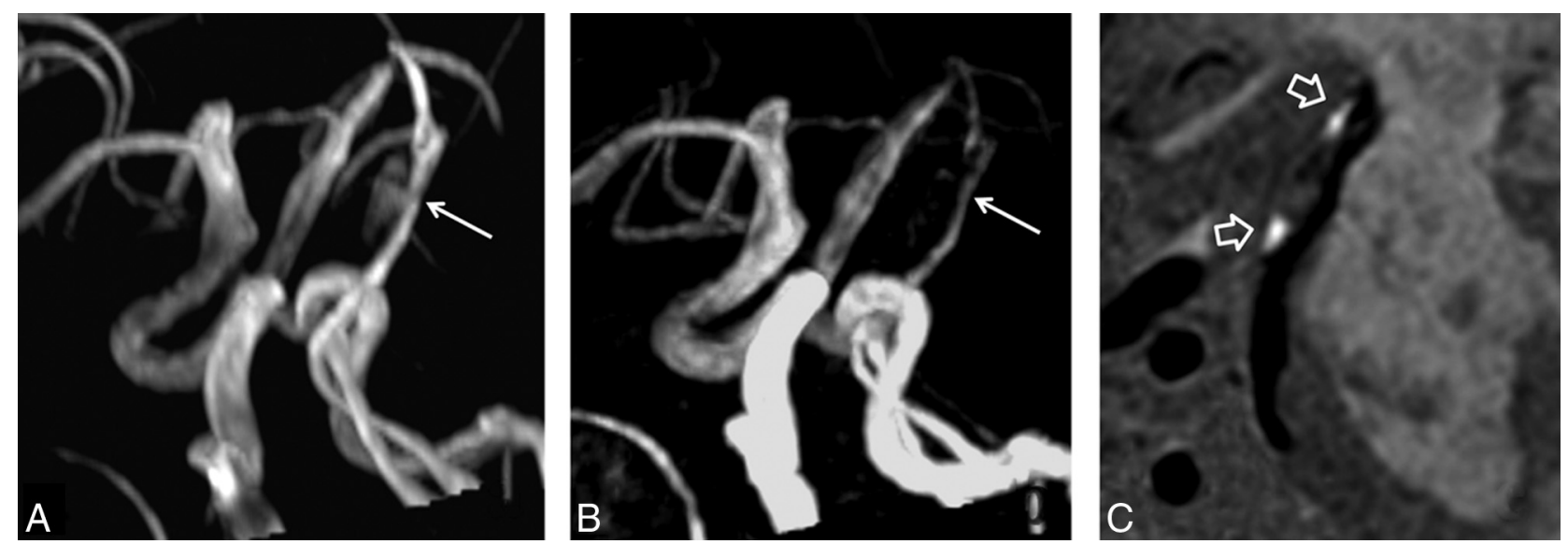

FIG 1. Excellent agreement between SNAP imaging and TOF MRA in measuring stenosis. Mild stenosis in the basilar artery is determined by MRA images derived from SNAP imaging $(A)$, which corresponds to the TOF MRA image $(B)$. The hyperintense signal on the SNAP vessel wall image $(C)$ indicates intramural hematoma.
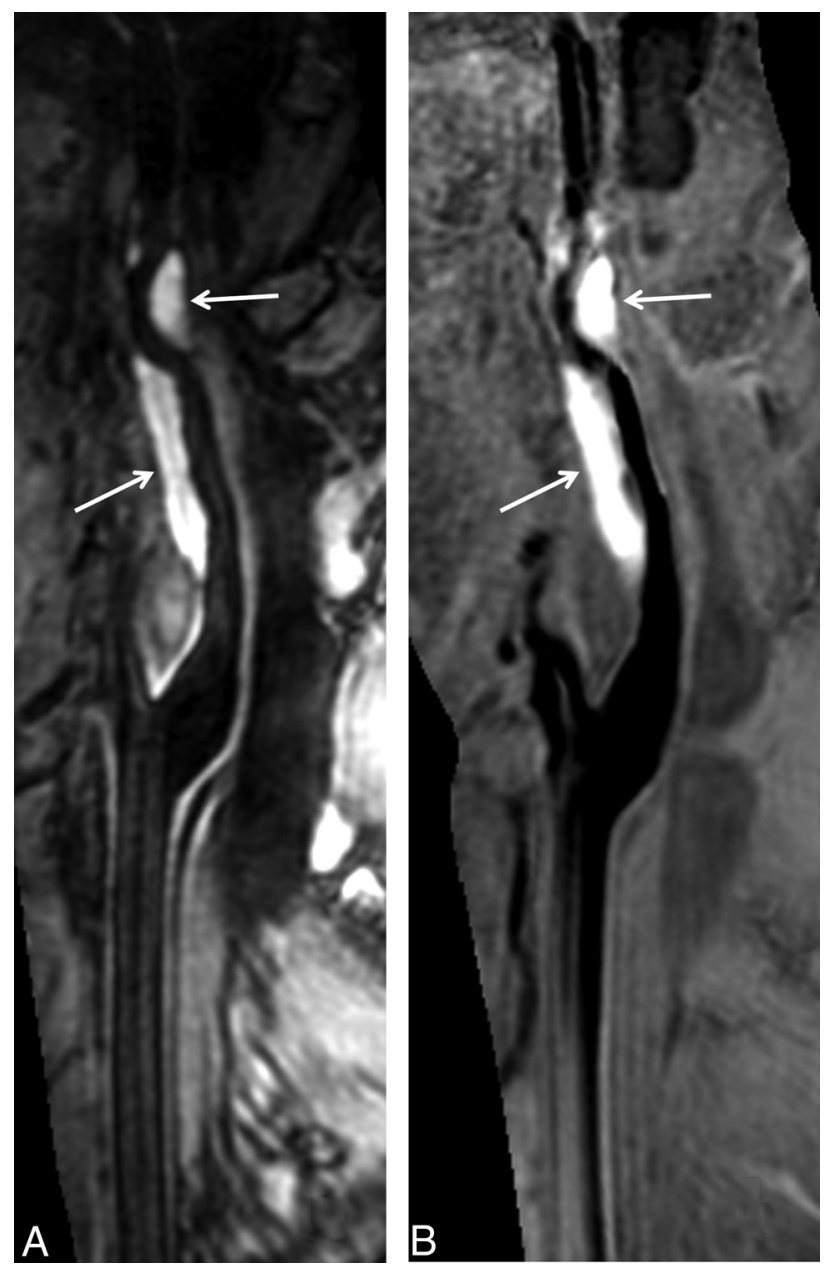

FIG 2. SNAP imaging depicts intramural hematoma. The intramural hematoma in the internal carotid artery appears hyperintense on both 3D MERGE ( $A$, white arrows) and SNAP images $(B$, white arrows).

\section{DISCUSSION}

This study investigated the feasibility of 3D SNAP imaging in characterizing CCAD. We found that 3D SNAP imaging showed excellent agreement with multisequence 3D MR imaging in measuring luminal stenosis and identification of IMH. In addition, $20.8 \%$ (5/24) of patients developed no or mild stenosis and $79.2 \%(19 / 24)$ of patients had IMH, indicating the importance of delineating wall changes by vessel wall imaging in the diagnosis of dissection. Because SNAP imaging provides noncontrast MRA and vessel wall images simultaneously with large longitudinal coverage and much shorter scanning time, it might be an alternative, time-efficient diagnostic tool for characterization of CCAD compared with multisequence 3D MR imaging.

We found that noncontrast MRA images derived from SNAP imaging showed excellent agreement with 3D TOF MRA in evaluating the luminal stenosis in patients with craniocervical artery dissection. Our findings are consistent with those in previous studies. Wang et al ${ }^{14}$ demonstrated that there was significant correlation between SNAP imaging and TOF MRA in the quantification of the lumen area of carotid arteries (intraclass correlation coefficient $=0.96 ; 95 \%$ CI, 0.94-0.97). Previous study ${ }^{14}$ has also shown that the SNAP image will generate a smaller lumen size compared with TOF MRA when the lumen area is $<20 \mathrm{~mm}^{2}$, indicating that it may overestimate the degree of stenosis at the arterial segment with a naturally small lumen size or severe luminal narrowing. Similarly, the luminal stenosis measured by SNAP images was overestimated compared with 3D TOF MRA in 3 arteries. In addition, the SNAP technique does not require contrast injection, so it can be used in patients with poor intravenous access or contraindications to gadolinium contrast administration (eg, pregnancy or renal failure). Our results indicate that noncontrast MRA derived from SNAP imaging might be an alternative approach for assessing the luminal changes in arterial dissection.

All the IMHs of this study population were successfully detected by 3D SNAP imaging. The IMH exhibited hyperintense signals on SNAP images, indicating the component with a short T1. It has been shown that the SNAP imaging sequence increased the contrast between hemorrhage and the vessel wall by another $35 \%$, which allows more detection of carotid intraplaque hemorrhage compared with the widely used 3D magnetization prepared rapid acquisition of gradient echo sequence. ${ }^{14}$ In previous studies, 

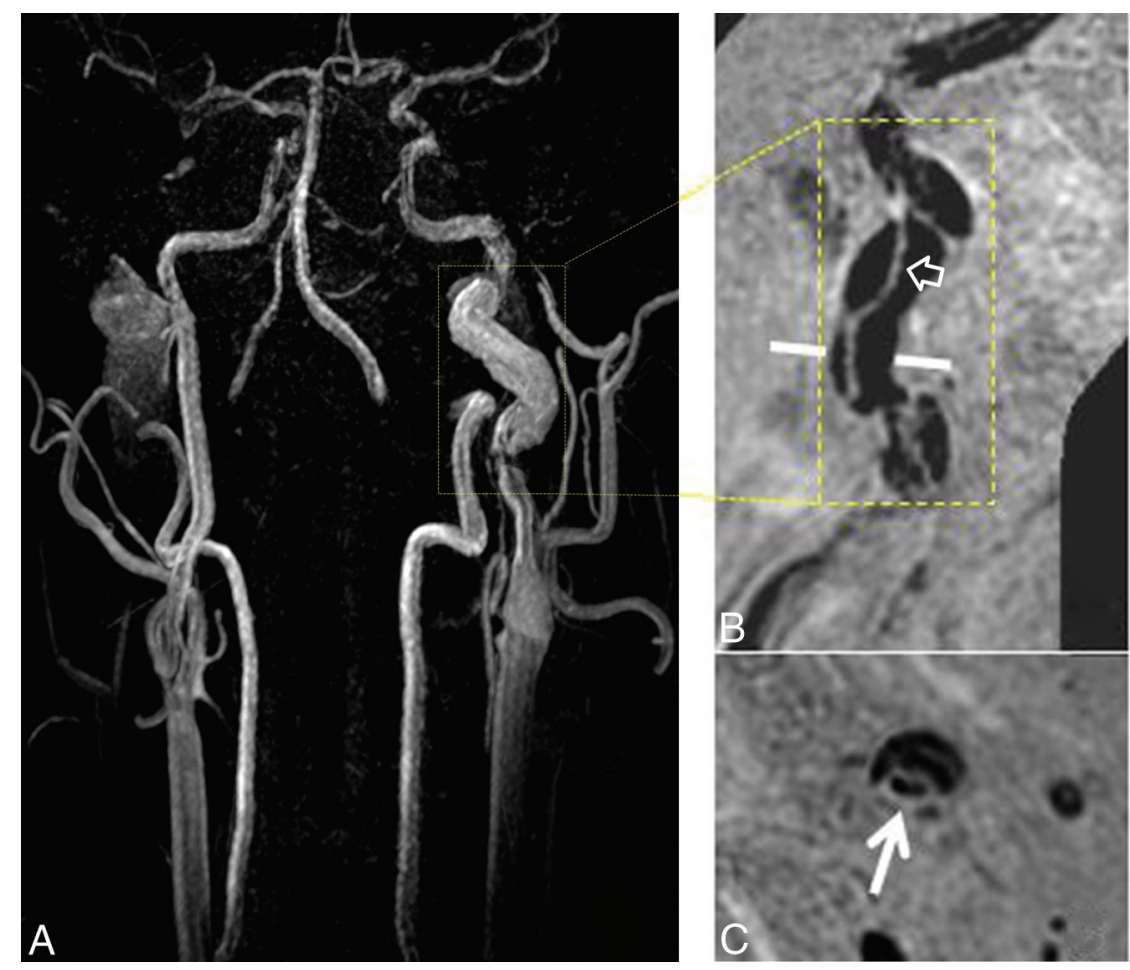

FIG 3. SNAP imaging delineates the intimal flaps. The SNAP MRA image $(A)$ shows a dilated lumen (pseudoaneurysm) in the internal carotid artery. The intimal flaps (hollow arrow) and double lumen (white arrow) are noted on vessel wall images derived from SNAP imaging in the coronal (B) and axial $(C)$ views after MPR reconstruction. The white line on the coronal view indicates the location of the axial view acquisition. the IMH was evaluated by 2D T1weighted MR imaging with fat saturation. ${ }^{11-13}$ However, acquisition of $2 \mathrm{D}$ T1-weighted images is time-consuming and has limited longitudinal coverage. Recently, the 3D black-blood T1weighted imaging technique has been used to detect IMH in patients with arterial dissection. ${ }^{19-21}$ Taking advantage of large coverage, black-blood effect, and high spatial resolution, 3D blackblood imaging sequences seem to be a substitute for 2D imaging techniques. Similar to the 3D T1-weighted imaging sequences, 3D SNAP imaging techniques enable fast and high-resolution imaging with large longitudinal coverage of up to $250 \mathrm{~mm}$. Benefiting from the large coverage of SNAP imaging, the extent of IMHs, particularly those involving multiple vascular beds, can be delineated.

In the present study, a substantial number of patients only had intimal flaps and double lumen without evidence of luminal stenosis and IMH. The intimal flaps and double lumen were

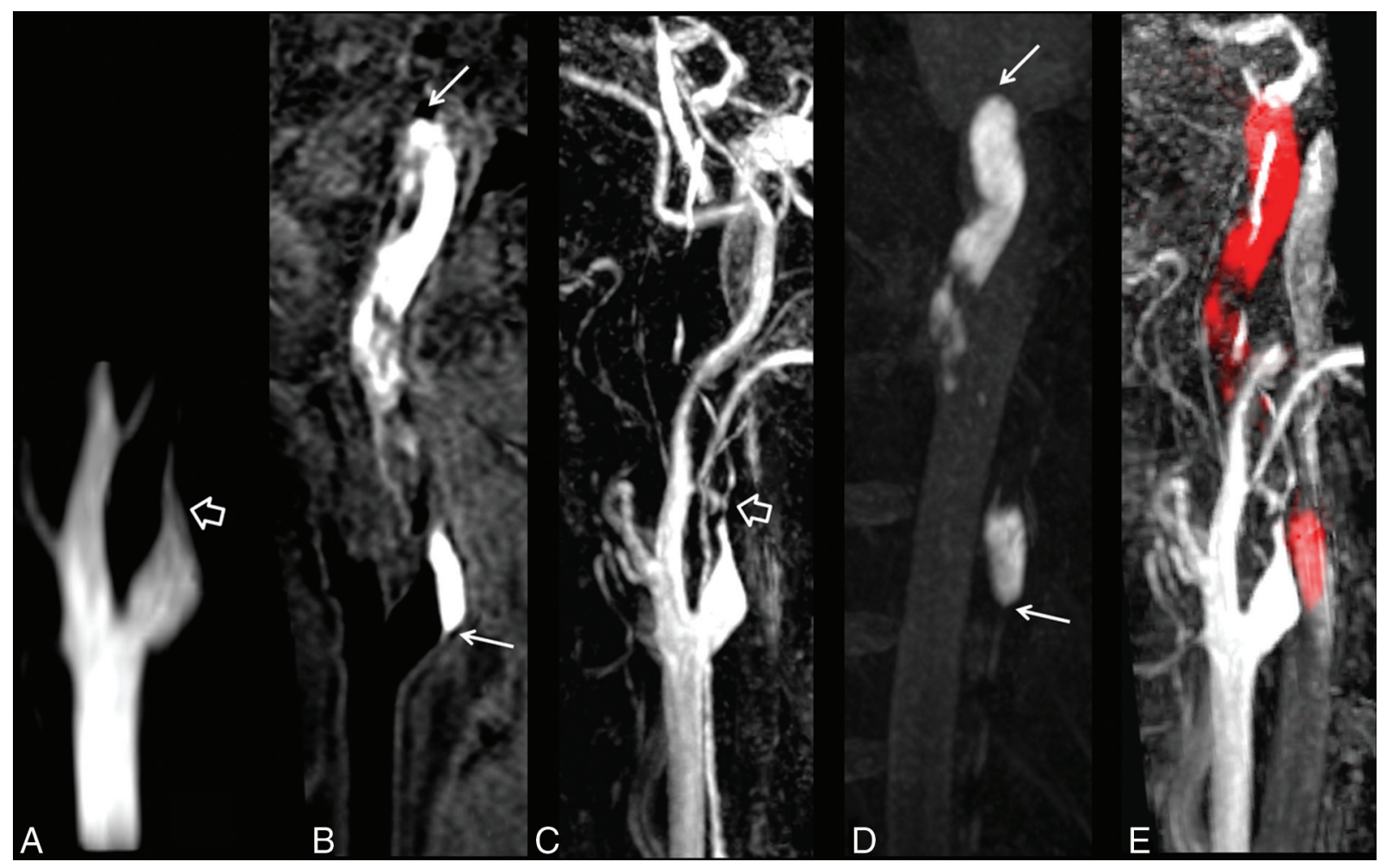

FIG 4. The luminal narrowing and intramural hematoma are jointly visualized in a single SNAP image. Total occlusion in the internal carotid artery is demonstrated by TOF MRA ( $A$, hollow arrow) and SNAP MRA (C, hollow arrow). A vessel wall image (B) derived from SNAP imaging shows a hyperintense lesion in the vessel wall (white arrows), indicating intramural hematoma. The intramural hematoma is well-depicted by maximum intensity projection of SNAP images $(D)$. The MRA and vessel wall images derived from SNAP imaging are naturally registered to jointly visualize luminal narrowing and intramural hematoma in red $(E)$ after color-coded processing. 
successfully depicted on the vessel wall images derived from SNAP imaging in this study. Similar to luminal narrowing and $\mathrm{IMH}$, these characteristics are important evidence for the diagnosis of dissection. It has been shown that these patterns exist when patients developed complete recanalization after dissection, but they were only seen in approximately $10 \%$ of cases. ${ }^{22}$ Our findings suggest that SNAP imaging might be a useful tool for comprehensive evaluation of CCAD.

For the diagnosis of arterial dissection, SNAP imaging seems to be a time-efficient approach that saves nearly $50 \%$ of scanning time compared with the recommended multisequence imaging protocol with 2 separate sequences (MRA and MR vessel wall imaging). This difference is because the 3D SNAP imaging can provide noncontrast MRA and vessel wall images simultaneously in a single scan in a much shorter scanning time. On the other hand, the MRA and vessel wall images generated from SNAP imaging are intrinsically registered by avoiding the misregistration between images acquired from 2 independent sequences. SNAP imaging enables joint visualization of the lumen and arterial wall changes at a single image. The assessment of the location of the relationship between the lumen and vessel wall in arteries with dissection might be useful for treatment planning of revascularization. Although SNAP imaging is capable of identifying CCAD comprehensively, multisequence MR imaging techniques might be needed in some complicated cases.

In this study, a custom-designed 36-channel coil was used to acquire intracranial and extracranial vessel wall images simultaneously. Compared with a commercialized neurovascular coil, this coil allows large-coverage MR imaging with a sufficient signal-to-noise ratio due to the dedicated coil elements for extracranial carotid arteries. The design of this 36-channel coil might be desirable for assessment of the arterial dissection, particularly for lesions involving a large extent of vessel wall or multiple vessels (carotid arteries and vertebral arteries) simultaneously.

Our study has several limitations. First, the sample size of this study was small, and all patients were suspected of having dissection. Future studies with a large sample size and a broad spectrum of disease should be conducted. Second, to determine the feasibility of SNAP imaging in the detection of IMH, the 3D MERGE imaging sequence was considered as a reference technique. This may decrease the specificity of identifying IMH because 3D MERGE introduces both T1- and T2-contrast-weighting during imaging. Finally, the signal pattern of IMH on SNAP imaging was obtained at only 1 time point. Previous studies reported that the signal of IMH will change with time on MR vessel wall imaging. ${ }^{13,22}$ To evaluate the usefulness of SNAP imaging in the characterization of CCAD, further prospective studies with larger sample sizes are warranted.

\section{CONCLUSIONS}

We found 3D SNAP imaging to have excellent agreement with multisequence MR imaging in the assessment of luminal stenosis and intramural hematoma in patients with CCAD. Our findings suggest that benefiting from fast imaging with large longitudinal coverage, 3D SNAP imaging might be an alternative, time-efficient diagnostic tool for CCAD compared with multisequence MR imaging.

Disclosures: Jinnan Wang_UNRELATED: Employment: Philips Research North America, Comments: full-time employee; Huijun Chen—RELATED: Grant: National Natural Science Foundation of China (research grants); UNRELATED: Philips Healthcare (research grants). ${ }^{*}$ Chun Yuan—RELATED: Grant: Philips Healthcare, ${ }^{*}$ National Institutes of Health, ${ }^{*}$ UNRELATED: Consultancy: Philips Healthcare, Comments: I am a member of the Radiology Medical Advisory Network of Philips Healthcare; Patents (planned, pending or issued): A patent was filed for the imaging technique used in this study. * Money paid to the institution.

\section{REFERENCES}

1. Leys D, Bandu L, Hénon H, et al. Clinical outcome in 287 consecutive young adults (15 to 45 years) with ischemic stroke. Neurology 2002;59:26-33

2. Ducrocq X, Lacour JC, Debouverie M, et al. Cerebral ischemic accidents in young subjects: a prospective study of 296 patients aged 16 to 45 years. Rev Neurol 1999;155:575-82

3. Schievink WI. Spontaneous dissection of the carotid and vertebral arteries. N Engl J Med 2001;344:898-906

4. Benninger DH, Georgiadis D, Kremer C, et al. Mechanism of ischemic infarct in spontaneous carotid dissection. Stroke 2004; 35:482-85

5. Dziewas R, Konrad C, Dräger B, et al. Cervical artery dissectionclinical features, risk factors, therapy and outcome in 126 patients. J Neurol 2003;250:1179-84

6. Biousse V, D'Anglejan-Chatillon J, Touboul PJ, et al. Time course of symptoms in extracranial carotid artery dissections: a series of $\mathbf{8 0}$ patients. Stroke 1995;26:235-39

7. Sturzenegger M, Mattle HP, Rivoir A, et al. Ultrasound findings in carotid artery dissection: analysis of $\mathbf{4 3}$ patients. Neurology 1995;45:691-98

8. Leclerc X, Godefroy O, Salhi A, et al. Helical CT for the diagnosis of extracranial internal carotid artery dissection. Stroke 1996; 27:461-66

9. Lévy C, Laissy JP, Raveau V, et al. Carotid and vertebral artery dissections: three-dimensional time-of-flight MR angiography and MR imaging versus conventional angiography. Radiology 1994;190:97-103

10. Debette S, Leys D. Cervical-artery dissections: predisposing factors, diagnosis, and outcome. Lancet Neurol 2009;8:668-78

11. Ozdoba C, Sturzenegger M, Schroth G. Internal carotid artery dissection: MR imaging features and clinical-radiologic correlation. Radiology 1996;199:191-98

12. Rodallec MH, Marteau V, Gerber S, et al. Craniocervical arterial dissection: spectrum of imaging findings and differential diagnosis. Radiographics 2008;28:1711-28

13. Goldberg HI, Grossman RI, Gomori JM, et al. Cervical internal carotid artery dissecting hemorrhage: diagnosis using MR. Radiology 1986;158:157-61

14. Wang J, Börnert P, Zhao H, et al. Simultaneous noncontrast angiography and intraplaque hemorrhage (SNAP) imaging for carotid atherosclerotic disease evaluation. Magn Reson Med 2013;69:337-45

15. Patel RR, Adam R, Maldjian C, et al. Cervical carotid artery dissection: current review of diagnosis and treatment. Cardiol Rev 2012;20:145-52

16. Wang X, Li R, Hayes $C$, et al. A new designed 36-channel neurovascular coil at 3T. In: Proceedings of the Annual Meeting of the International Society of Magnetic Resonance in Medicine, Melbourne, Australia. May 5-11, 2012;21:2787 
17. Balu N, Yarnykh VL, Chu B, et al. Carotid plaque assessment using fast 3D isotropic resolution black-blood MRI. Magn Reson Med 2011;65:627-37

18. North American Symptomatic Carotid Endarterectomy Trial Collaborators. Beneficial effect of carotid endarterectomy in symptomatic patients with high-grade carotid stenosis. $N$ Engl J Med 1991;325:445-53

19. Cuvinciuc V, Viallon M, Momjian-Mayor I, et al. 3D fat-saturated T1 SPACE sequence for the diagnosis of cervical artery dissection. Neuroradiology 2013;55:595-602
20. Edjlali M, Roca P, Rabrait C, et al. 3D fast spin-echo T1 black-blood imaging for the diagnosis of cervical artery dissection. AJNR Am J Neuroradiol 2013;34:E103-06

21. Takano K, Yamashita S, Takemoto K, et al. MRI of intracranial vertebral artery dissection: evaluation of intramural haematoma using a black blood, variable-flip-angle 3D turbo spin-echo sequence. Neuroradiology 2013;55:845-51

22. Flis CM, Jager HR, Sidhu PS. Carotid and vertebral artery dissections: clinical aspects, imaging features and endovascular treatment. Eur Radiol 2007;17:820-34 